\title{
An intrusion layer in stationary incompressible fluids Part 2: A solitary wave
}

\author{
LAWRENCE K. FORBES ${ }^{1}$ and GRAEME C. HOCKING ${ }^{2}$ \\ ${ }^{1}$ School of Mathematics and Physics, University of Tasmania, Hobart 7001, Tasmania, Australia \\ email: larry.forbes@utas.edu.au \\ ${ }^{2}$ School of Mathematics and Statistics, Division of Science, Murdoch University, Murdoch 6150, Western Australia
}

(Received 30 November 2005; revised 1 June 2006)

\begin{abstract}
The propagation of a solitary wave in a horizontal fluid layer is studied. There is an interfacial free surface above and below this intrusion layer, which is moving at constant speed through a stationary density-stratified fluid system. A weakly nonlinear asymptotic theory is presented, leading to a Korteweg-de Vries equation in which the two fluid interfaces move oppositely. The intrusion layer solitary wave system thus forms a widening bulge that propagates without change of form. These results are confirmed and extended by a fully nonlinear solution, in which a boundary-integral formulation is used to solve the problem numerically. Limiting profiles are approached, for which a corner forms at the crest of the solitary wave, on one or both of the interfaces.
\end{abstract}

\section{Introduction}

This is the second in a series of two papers that consider a moving intrusion layer in otherwise stationary stratified fluid. Such situations may arise under a variety of different circumstances. They may occur in reservoirs when river water flows in from a tributary, sinks down to its neutrally buoyant level, and then spreads horizontally. In such a flow, the layer of intruding water may be separated from the lighter fluid above it and the heavier water below by two horizontal interfaces.

Benjamin [1] has given a discussion of general two-layer intrusion currents under gravity, and has concluded that the advancing head of the intrusion layer must form a breaking wave as it propagates. An experimental investigation of this situation for a three-layer system was described by Mehta et al. [2]. Each layer had constant density and only the middle fluid was in motion, with the top and bottom layers stationary. They showed that the intrusion initially propagated along the middle layer as a bulbous head, leaving behind it a train of waves on each interface. Detailed photographs were included. Similar experimental work has been presented by Sutherland et al. [3] and Manins [4].

Withdrawal from the middle layer of a three-fluid system has been investigated by Wen \& Ingham [5], and they have shown that both the upper and the lower interfaces may be drawn towards the sink in the middle layer, to form a system possessing two inward-facing cusps. Qualitatively similar flows are also known to exist in ground-water flows, and have been computed by Papatzacos \& Gustafson [6], for example. 
If the fluid above and below the intrusion layer is at rest, then a train of waves may be formed in this moving middle layer, and involves waves on both the upper and lower interfaces. This was apparently first recognized by Lord Rayleigh, and has been discussed by Lamb [7, art. 232]. There it was assumed that all three fluid layers had the same density. This linearized solution was generalized in Paper 1 [8] to allow all three fluid layers to have different densities, and it was confirmed there also that two different propagation modes are possible. There is a high-speed type in which both interfaces move in phase, and a low-speed branch for which the two interfaces move oppositely.

Similar intrusion currents may also be formed in the atmosphere. Flynn \& Sutherland [9] have described how the anvil cloud of a thunderstorm may form an intrusion layer at about the level of the tropopause, for example. Two-layer atmospheric intrusion flows can arise when a heavier layer penetrates beneath a lighter one, and such a process is believed to be involved in the remarkable 'Morning Glory' phenomenon in the Gulf of Carpentaria in the far north of Australia, as described by Clarke et al. [10]. A mathematical model of this phenomenon was presented by Forbes \& Belward [11], assuming the presence of a solitary wave on the interface between the two layers. Similar kinds of flows may be produced pyroclastically due to volcanic eruptions, as discussed by Nield \& Woods [12]. Three-layer atmospheric intrusion flows can also occur above rivers, and the 'Bridgewater Gerry' in Hobart in Tasmania is a striking example of this effect; see Figure 13 in Paper 1 [8], for example.

In this second paper, an analysis is undertaken of solitary waves propagating along the middle layer of a three-fluid system. As in Paper 1 [8], both the upper and lower fluids are at rest and are of infinite depth. The theory of solitary waves has a long and distinguished history, and an overall review of the classical work in this field has been given by Miles [13]. One of the earlier numerical computations of steep solitary waves was the work of Yamada [14], who used a conformal mapping technique that anticipated much modern analysis. He concluded that the highest solitary wave encloses a sharp corner at its crest. This work was extended by Hunter \& Vanden-Broeck [15] using both a conformal mapping approach with a series expansion and an integral-equation technique. They computed solitary waves accurately up to the highest one enclosing the Stokes angle $120^{\circ}$ at the crest.

Recently, solitary waves have been computed in more complex environments, such as between two homogeneous fluids [16], in the presence of constant vorticity [11, 17], and including the effects of surface tension [18, 19], for example. Laget \& Dias [20] have shown that there are in fact two types of solitary wave when surface tension is included. Their paper also includes a discussion of the possible configuration of the limiting wave, which may involve a corner at the crest with enclosed angle $120^{\circ}$, a flat broad crest or else overhanging sections in the wave profile. Results for three-layer fluids, with mean flow in each of the fluid layers, have been obtained numerically by Rusås \& Grue [21], and it was seen in that problem that solitary waves of both elevation and depression could be computed. For elevation solitary waves, profiles with extreme overhanging sections were obtained. Forced solitary waves created by a bottom obstruction have been obtained by Dias \& Vanden-Broeck [22], using an integral-equation technique for the fully nonlinear problem in addition to a weakly nonlinear theory for moderate disturbances. That approach corresponds reasonably closely to the viewpoint of the present paper. 


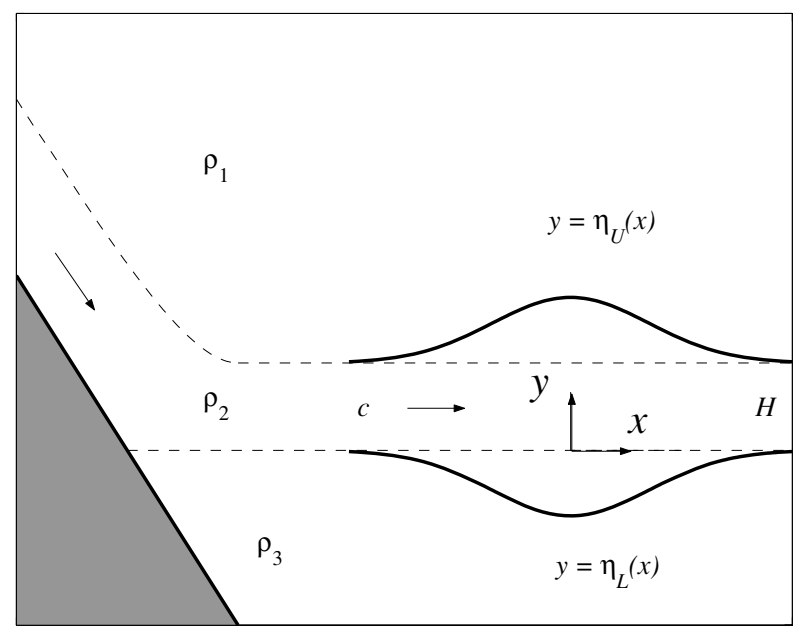

FIGURE 1. Illustration of an intrusion layer created when water enters a reservoir. The fluid of intermediate density $\rho_{2}$ initially flows down into the reservoir before moving horizontally at its neutrally buoyant height. A solitary wave is established in the moving middle layer, with its two interfaces bulging outward, as sketched.

The governing equations for this problem are reviewed in $\S 2$, and the results for the (linearized) small amplitude solution for waves of finite wavelength are summarized from Paper 1 [8]. In $\S 3$, the weakly nonlinear theory is developed, leading to a Korteweg-de Vries equation for the interface shapes. The solitary wave is obtained as the homoclinic orbit of this dynamical system, and also yields a speed-amplitude relation for solitary waves of moderate height. The numerical solution algorithm for the fully nonlinear problem is outlined in $\S 4$, and is based on the use of integral equations in the physical plane of the solution variables. Numerical results are presented and discussed in $\S 5$, and the paper concludes with a discussion in $\S 6$.

\section{The governing equations}

Consider an unbounded stratified fluid subject to the downward acceleration $g$ of gravity. It is assumed that the fluid consists of three horizontal layers, each separated from the other by a sharp interface. There are thus two horizontal interfaces, and a cartesian coordinate system is located on the lower interface, with the $y$-axis pointing vertically (in the opposite direction to the acceleration of gravity). The top, middle and bottom fluid layers have densities $\rho_{1}, \rho_{2}$ and $\rho_{3}$, respectively, and it is a physical requirement that $\rho_{1}<\rho_{2}<\rho_{3}$ for stability. The height of the middle layer is $H$.

The top and bottom layers are assumed to be stationary and of infinite depth, but the middle layer (of density $\rho_{2}$ ) is moving with speed $c$. This is therefore an intrusion layer of thickness $H$ that is entering an otherwise stationary stratified fluid. It is assumed that the flow is two-dimensional, with horizontal and vertical velocity components $u$ and $v$ in the moving middle fluid layer.

A sketch of the three-layer intrusion system is given in Figure 1, illustrating the situation that might be encountered in a reservoir. The moving middle layer of intermediate density 
$\rho_{2}$ is created far to the left of the picture by a flow moving down the reservoir side wall, and detaching from the wall and entering the reservoir at the height of its neutral buoyancy. A stationary solitary wave is formed at some position, and the $y$-axis of the cartesian coordinate system is located at its crests, as indicated. The upper and lower interfaces have equations $y=\eta_{U}(x)$ and $y=\eta_{L}(x)$.

Dimensionless coordinates and variables are now defined, and used throughout the remainder of this paper. All lengths are scaled relative to the mean depth $H$ of the middle layer, and all speeds are measured relative to the mean speed $c$ of that flowing intrusion layer. In these dimensionless variables, the middle layer therefore has unit depth and speed far upstream and downstream of the solitary wave. The fluid system is assumed to be ideal in the sense that it is incompressible, inviscid and therefore flows irrotationally; a velocity potential $\phi$ thus exists for the moving middle layer, and has been made dimensionless with respect to the product $c H$. The solitary wave is then determined by the three dimensionless parameters:

$$
F=\frac{c}{\sqrt{g H}} \quad \gamma_{1}=\frac{\rho_{1}}{\rho_{2}} \quad \gamma_{3}=\frac{\rho_{3}}{\rho_{2}}
$$

as in Paper 1 [8]. The first of these parameters $F$ is the Froude number for the moving middle intrusion layer, and represents a ratio of its speed to a characteristic speed dependent on its depth. The two constants $\gamma_{1}<1$ and $\gamma_{3}>1$ are density ratios of the stationary top and bottom fluids relative to that of the middle intrusion layer. It will also be convenient to define a fourth parameter,

$$
A_{T}=\eta_{L}(\infty)-\eta_{L}(0)
$$

which is a measure of the amplitude of the solitary wave system, as measured in terms of the behaviour of the lower interface.

Within the moving middle layer, the two velocity components are given in terms of the derivatives $u=\partial \phi / \partial x$ and $v=\partial \phi / \partial y$ of the velocity potential $\phi$, which in turn satisfies Laplace's equation

$$
\frac{\partial^{2} \phi}{\partial x^{2}}+\frac{\partial^{2} \phi}{\partial y^{2}}=0 \quad \text { in } \eta_{L}(x)<y<\eta_{U}(x) .
$$

On the upper interface, there is a kinematic condition

$$
v=u \frac{d \eta_{U}}{d x} \quad \text { on } y=\eta_{U}(x)
$$

representing the fact that the fluid cannot cross this boundary, and a dynamic condition

$$
\frac{1}{2} F^{2}\left(u^{2}+v^{2}\right)+\left(1-\gamma_{1}\right)\left(\eta_{U}-1\right)=\frac{1}{2} F^{2} \quad \text { on } y=\eta_{U}(x)
$$

which expresses the continuity of pressure across this interface. Similarly, the kinematic condition on the lower interface is

$$
v=u \frac{d \eta_{L}}{d x} \quad \text { on } y=\eta_{L}(x)
$$


and the dynamic condition takes the form

$$
\frac{1}{2} F^{2}\left(u^{2}+v^{2}\right)-\left(\gamma_{3}-1\right) \eta_{L}=\frac{1}{2} F^{2} \quad \text { on } y=\eta_{L}(x)
$$

Solitary waves in this system are thus obtained as solutions of equations (2.2)-(2.7).

A linearized solution to equations (2.3)-(2.7), for periodic waves of wavelength $\lambda$ and wavenumber $k=2 \pi / \lambda$, is discussed in Paper 1 [8]. It is shown there that the Froude number $F$ is obtained in terms of the wavenumber $k$ by means of the dispersion relation:

$$
2 F^{2} k \sinh k=\left(\gamma_{3}-\gamma_{1}\right) \cosh k \pm \sqrt{\left(\gamma_{3}-\gamma_{1}\right)^{2} \cosh ^{2} k-4\left(\gamma_{3}-1\right)\left(1-\gamma_{1}\right) \sinh ^{2} k}
$$

The argument of the square root term in this expression (2.8) is always positive, and both of the two signs on the right hand side yield positive values for the Froude number $F$. There are thus two possible propagation modes for periodic intrusion waves. When the plus sign is chosen in (2.8), a higher speed solution is obtained, in which both interfaces are in phase. The minus sign in (2.8) gives rise to a second propagation mode at lower speed, for which the two interfaces are of opposite phase.

Solitary waves may be thought of as bifurcating from the linearized solution (2.8) in the limit of infinite wavelength, $k \rightarrow 0$. This limit may be investigated using Taylor series expansions in (2.8), and readily shows that a finite Froude number limit is not obtained when the plus sign is chosen. Solitary waves are therefore not possible for this higher-speed propagation mode with the two interfaces in phase.

However, the lower-speed wave type, obtained with the negative sign in equation (2.8), yields the finite limit

$$
F \rightarrow \sqrt{\frac{\left(1-\gamma_{1}\right)\left(\gamma_{3}-1\right)}{\left(\gamma_{3}-\gamma_{1}\right)}}
$$

as $k \rightarrow 0$. Solitary waves are thus only possible for the one propagation mode in which the two interfaces are in anti-phase, and they bifurcate from the trivial uniform-flow solution at the value of Froude number given by equation (2.9).

\section{Weakly nonlinear theory}

This section derives the solution for the double-interface solitary wave in the weakly nonlinear approximation, essentially following the development outlined in Wehausen \& Laitone [23, section 10]. To begin, new scaled variables are introduced, according to the relations

$$
\bar{x}=x \sqrt{\epsilon} ; \quad \bar{y}=y ; \quad \bar{u}=u ; \quad \bar{v}=v \sqrt{\epsilon} ; \quad \bar{p}=p .
$$

Here, the square root of the parameter $\epsilon$ is intended to represent the ratio of vertical to horizontal length scales, and is assumed to be small. The variable $p$ denotes pressure in the middle layer.

The full set of governing equations is written in these scaled variables (3.1), in the moving intrusion layer $\bar{\eta}_{L}(\bar{x})<\bar{y}<\bar{\eta}_{U}(\bar{x})$. The continuity equation becomes

$$
\epsilon \frac{\partial \bar{u}}{\partial \bar{x}}+\frac{\partial \bar{v}}{\partial \bar{y}}=0
$$


and the condition of irrotational flow is

$$
\frac{\partial \bar{v}}{\partial \bar{x}}=\frac{\partial \bar{u}}{\partial \bar{y}}
$$

The horizontal and vertical components of the momentum equation take the forms

$$
\epsilon\left(F^{2} \bar{u} \frac{\partial \bar{u}}{\partial \bar{x}}+\frac{\partial \bar{p}}{\partial \bar{x}}\right)+F^{2} \bar{v} \frac{\partial \bar{u}}{\partial \bar{y}}=0
$$

and

$$
\epsilon\left(F^{2} \bar{u} \frac{\partial \bar{v}}{\partial \bar{x}}+\frac{\partial \bar{p}}{\partial \bar{y}}+1\right)+F^{2} \bar{v} \frac{\partial \bar{v}}{\partial \bar{y}}=0
$$

respectively.

On the upper interface $\bar{y}=\bar{\eta}_{U}(\bar{x})$, the kinematic condition (2.4) becomes

$$
\bar{v}=\epsilon \bar{u} \frac{d \bar{\eta}_{U}}{d \bar{x}}
$$

and the dynamic condition (2.5) is replaced with the equivalent statement

$$
\bar{p}=\bar{p}_{\infty}-\gamma_{1} \bar{y} .
$$

Similarly, the kinematic and dynamic conditions (2.6) and (2.7) on the lower interface $\bar{y}=\bar{\eta}_{L}(\bar{x})$ become

and

$$
\bar{v}=\epsilon \bar{u} \frac{d \bar{\eta}_{L}}{d \bar{x}}
$$

$$
\bar{p}=\bar{p}_{\infty}+1-\gamma_{1}-\gamma_{3} \bar{y}
$$

respectively.

The solution variables and the Froude number are expanded as regular perturbation series in $\epsilon$, and take the forms

$$
\begin{aligned}
\bar{u} & =\bar{u}_{0}+\epsilon \bar{u}_{1}+\epsilon^{2} \bar{u}_{2}+\epsilon^{3} \bar{u}_{3}+\mathcal{O}\left(\epsilon^{4}\right) \\
\bar{v} & =\bar{v}_{0}+\epsilon \bar{v}_{1}+\epsilon^{2} \bar{v}_{2}+\epsilon^{3} \bar{v}_{3}+\mathcal{O}\left(\epsilon^{4}\right) \\
\bar{\eta}_{U} & =\bar{H}_{0}+\epsilon \bar{H}_{1}+\epsilon^{2} \bar{H}_{2}+\epsilon^{3} \bar{H}_{3}+\mathcal{O}\left(\epsilon^{4}\right) \\
\bar{\eta}_{L} & =\bar{L}_{0}+\epsilon \bar{L}_{1}+\epsilon^{2} \bar{L}_{2}+\epsilon^{3} \bar{L}_{3}+\mathcal{O}\left(\epsilon^{4}\right) \\
\bar{p} & =\bar{P}_{0}+\epsilon \bar{P}_{1}+\epsilon^{2} \bar{P}_{2}+\epsilon^{3} \bar{P}_{3}+\mathcal{O}\left(\epsilon^{4}\right) \\
\bar{F}^{2} & =\bar{G}_{0}+\epsilon \bar{G}_{1}+\epsilon^{2} \bar{G}_{2}+\epsilon^{3} \bar{G}_{3}+\mathcal{O}\left(\epsilon^{4}\right) .
\end{aligned}
$$

It is evident from the original non-dimensionalization of this problem that it is necessary to set $\bar{H}_{0}=1$ and $\bar{L}_{0}=0$. The expansions (3.10) are substituted into the full system (3.2)(3.9) of partial differential equations and boundary conditions, and terms are collected at each order in the parameter $\epsilon$.

At the zeroth order in $\epsilon$, it is found that

$$
\bar{v}_{0}=0 \quad \text { and } \quad \bar{u}_{0}=\bar{u}_{0}(\bar{x}) .
$$


The first-order equations then show that

$$
\begin{aligned}
\bar{u}_{0} & =1 \\
\bar{u}_{1} & =\bar{u}_{1}(\bar{x}) \\
\bar{v}_{1} & =0 \\
\bar{P}_{0}(\bar{y}) & =\bar{p}_{\infty}+1-\gamma_{1}-\bar{y}
\end{aligned}
$$

The equations at the second order in $\epsilon$ now show that

$$
\bar{G}_{0}=\frac{\left(1-\gamma_{1}\right)\left(\gamma_{3}-1\right)}{\left(\gamma_{3}-\gamma_{1}\right)} .
$$

This result (3.13) gives the Froude number at which the solitary wave bifurcates from uniform flow. It is identical to the result (2.9) obtained as the long-wavelength limit of the small-amplitude wave solution, for the branch of solutions with the two interfaces having opposite phase. In addition, the second-order solution yields the relations

$$
\begin{aligned}
& \bar{P}_{1}(\bar{x})=\left(1-\gamma_{3}\right) \bar{L}_{1}(\bar{x}), \\
& \bar{H}_{1}(\bar{x})=-\left(\frac{\gamma_{3}-1}{1-\gamma_{1}}\right) \bar{L}_{1}(\bar{x}), \\
& \bar{u}_{1}(\bar{x})=\left(\frac{\gamma_{3}-\gamma_{1}}{1-\gamma_{1}}\right) \bar{L}_{1}(\bar{x}),
\end{aligned}
$$

for the functions at first order in the expansions (3.10). In particular, the second equation in this group shows that the two interfaces must move oppositely to one another. Finally, the second-order solution gives the two velocity components at the second order in (3.10) in the forms

$$
\begin{aligned}
& \bar{v}_{2}(\bar{x}, \bar{y})=\left[1-\bar{y}\left(\frac{\gamma_{3}-\gamma_{1}}{1-\gamma_{1}}\right)\right] \frac{d \bar{L}_{1}}{d \bar{x}}, \\
& \bar{u}_{2}(\bar{x}, \bar{y})=\left[\bar{y}-\frac{1}{2} \bar{y}^{2}\left(\frac{\gamma_{3}-\gamma_{1}}{1-\gamma_{1}}\right)\right] \frac{d^{2} \bar{L}_{1}}{d \bar{x}^{2}}+g_{2}(\bar{x}),
\end{aligned}
$$

in which the function $g_{2}$ is so far arbitrary.

The remaining function $\bar{L}_{1}$ in the expressions (3.14) and (3.15) may be determined by making partial use of the equations to third order in $\epsilon$. After some considerable algebra and making use of (3.11)-(3.15), it is found that this function satisfies the third-order differential equation

$$
\frac{d^{3} \bar{L}_{1}}{d \bar{x}^{3}}-9 \beta_{1} \bar{L}_{1} \frac{d \bar{L}_{1}}{d \bar{x}}-3 \beta_{2} \bar{G}_{1} \frac{d \bar{L}_{1}}{d \bar{x}}=0 .
$$

In this expression, the two constants are defined to be

$$
\beta_{1}=\frac{\left(\gamma_{3}-\gamma_{1}\right)^{2}}{\left(1-\gamma_{1}\right)\left[\gamma_{3}-\gamma_{1}-3 \bar{G}_{0}\right]}, \quad \beta_{2}=\frac{\left(\gamma_{3}-\gamma_{1}\right)}{\bar{G}_{0}\left[\gamma_{3}-\gamma_{1}-3 \bar{G}_{0}\right]} .
$$

The constant $\bar{G}_{0}$ is the bifurcation value of the squared Froude number, as given in (3.13), and $\bar{G}_{1}$ is the first-order perturbation in (3.10). 


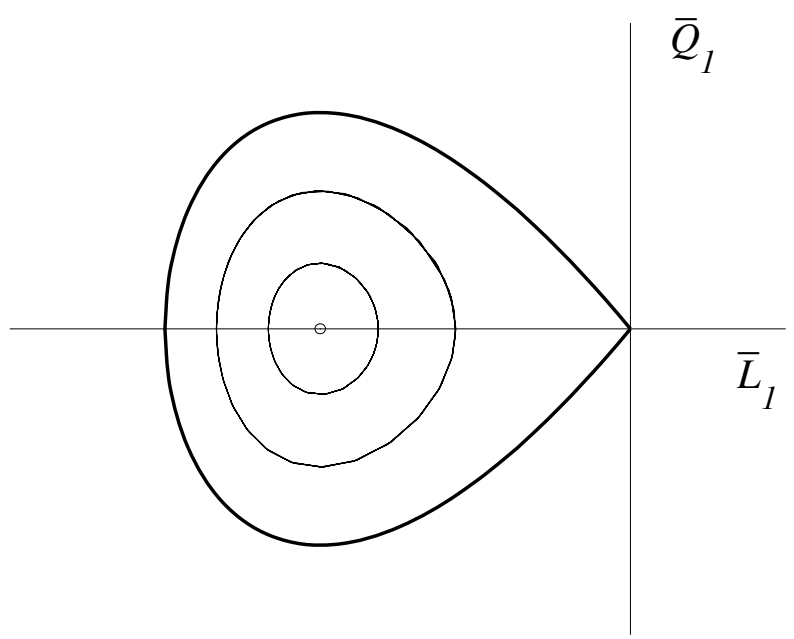

FIGURE 2. An illustration of the $\left(\bar{L}_{1}, \bar{Q}_{1}\right)$ phase plane, for the dynamical system (3.18). The soliton corresponds to the homoclinic orbit that passes into the saddle at the origin.

The expression (3.16) is a Korteweg-de Vries equation for the shape of the lower interface (see Wehausen \& Laitone [23] and Whitham [24]). From (3.14), the upper interface must satisfy the similar equation, although its sign is opposite. For the solitary wave, there is an immediate first integral of (3.16), under the requirement that the lower interface must become horizontal at infinity. The resulting second-order equation may then be written as the first-order system

$$
\begin{aligned}
\frac{d \bar{L}_{1}}{d \bar{x}} & =\bar{Q}_{1} \\
\frac{d \bar{Q}_{1}}{d \bar{x}} & =\frac{9}{2} \beta_{1} \bar{L}_{1}^{2}+3 \beta_{2} \bar{G}_{1} \bar{L}_{1} .
\end{aligned}
$$

This system (3.18) has the two stationary points

$$
\left(\bar{L}_{1}, \bar{Q}_{1}\right)=(0,0), \quad\left(-\frac{2 \beta_{2} \bar{G}_{1}}{3 \beta_{1}}, 0\right),
$$

and the fact that the solitary wave becomes flat at infinity means that the origin $(0,0)$ in equation (3.19) must be a saddle of the dynamical system (3.18). This is only possible if $\bar{G}_{1}>0$, which shows that the solitary wave moves faster than the speed given by the bifurcation value (2.9).

A phase-plane analysis of the dynamical system (3.18) shows that the solitary wave is the homoclinic orbit with equation

$$
\bar{Q}_{1}^{2}=3 \beta_{1} \bar{L}_{1}^{3}+3 \beta_{2} \bar{G}_{1} \bar{L}_{1}^{2},
$$

since it must pass into the origin. A sketch of the situation is given in Figure 2, in which the outermost loop corresponds to the solitary wave. The smaller inner loops surrounding the second of the stationary points in (3.19) represent periodic 'cnoidal' waves, and are similar 
to the anti-phase branch of periodic intrusion waves discussed in Paper 1 [8]. (However, cnoidal solutions to equations (3.18) are necessarily supercritical, as their Froude number must exceed the bifurcation value in equation (2.9). We do not discuss these further here.)

The system (3.18), (3.20) can be integrated in closed form, for the solitary wave, to give the famous 'sech-squared' profile. This is discussed in detail by Whitham [24], for example. (A summary of the various solitary wave theories is also given in the paper by Grue et al. [25].) In terms of the original variables obtained from (3.1) and (3.10), the lower and upper interfaces are predicted by this weakly nonlinear analysis to have the profiles

$$
\begin{aligned}
& \eta_{L}(x)=-A_{T} \operatorname{sech}^{2}\left((x / 2) \sqrt{3 \beta_{1} A_{T}}\right) \\
& \eta_{U}(x)=1+\frac{\gamma_{3}-1}{1-\gamma_{1}} A_{T} \operatorname{sech}^{2}\left((x / 2) \sqrt{3 \beta_{1} A_{T}}\right) .
\end{aligned}
$$

The horizontal velocity component is then determined with the aid of equation (3.14) to be

$$
u(x)=1-\frac{\gamma_{3}-\gamma_{1}}{1-\gamma_{1}} A_{T} \operatorname{sech}^{2}\left((x / 2) \sqrt{3 \beta_{1} A_{T}}\right) .
$$

In addition, the amplitude of the soliton, as defined by (2.2), is related to the Froude number $F$ by means of the equation

$$
F^{2}=\bar{G}_{0}+\left(\beta_{1} / \beta_{2}\right) A_{T},
$$

after the first-order term $\bar{G}_{1}$ has been eliminated using (3.10).

The limit $\gamma_{1} \rightarrow 0$ corresponds to the situation in which the top fluid has zero density; in that case, the upper interface becomes a free surface. The two constants in equations (3.17) become simply

$$
\beta_{1}=\frac{\left(\gamma_{3}\right)^{3}}{\left(\gamma_{3}\right)^{2}-3 \gamma_{3}+3}, \quad \beta_{2}=\frac{\beta_{1}}{\gamma_{3}-1},
$$

The interface shapes are as in equations (3.21), and the weakly nonlinear dispersion relation (3.23) has the simple form

$$
F^{2}=\left(\gamma_{3}-1\right)\left[1 / \gamma_{3}+A_{T}\right]
$$

Although (3.24) has a similar form to that of the solitary wave of depression summarized in Grue et al. [25], it is nevertheless a different solution, since in the present situation, the lowest fluid is stationary.

The result (3.21)-(3.23) thus represents the complete solitary wave solution in the weakly nonlinear analysis. It will be seen that it provides a good approximation for waves of moderate amplitude, and thus gives a good starting point for the fully nonlinear numerical solution of the next section.

\section{Numerical solution algorithm}

The numerical method used for the solution of the fully nonlinear system of equations (2.2)-(2.7) is discussed in this section. The formulation is based on the use of integral equations in the physical plane of the solution variables. 
Suppose that the complex position $z_{Q}=x_{Q}+i y_{Q}$ represents a point either on the upper interface $y=\eta_{U}(x)$ or on the lower interface $y=\eta_{L}(x)$. The complex function $f(z)=u(x, y)-i v(x, y)-1$ is analytic in the moving middle fluid layer, since the fluid is incompressible and flows irrotationally, and the velocity components satisfy the CauchyRiemann equations (from which (3.2) and (3.3) were derived). Furthermore, $f(z)$ vanishes far upstream and far downstream. It therefore follows from Cauchy's integral formula that

$$
\oint_{\Gamma} \frac{f\left(z_{P}\right)}{z_{P}-z_{Q}} d z_{P}=0
$$

In this expression, $P$ denotes a moveable point anywhere on the closed contour $\Gamma$. This contour is made up of the upper interface and the lower interface connected by vertical lines far upstream and far downstream; the point $z_{Q}$ on either interface is by-passed with a small semi-circular arc contained within the moving middle layer.

The contributions to the integral in equation (4.1) from the vertical lines at infinity are simply zero, since the function $f(z)$ vanishes there. Thus only the line integrals along the two interfaces and on the small semi-circle by-passing the point $Q$ on either interface need to be taken into account in equation (4.1). After a little algebra, and taking the imaginary part in (4.1), for point $Q$ on the upper interface it is found that

$$
\begin{aligned}
\pi(u-1)_{Q}= & C P V \int_{-\infty}^{\infty} \frac{T_{U}(P)\left(\eta_{U}(P)-\eta_{U}(Q)\right)+\delta x^{(-)} \eta_{U}^{\prime}(P)}{\left(\delta x^{(-)}\right)^{2}+\left(\eta_{U}(P)-\eta_{U}(Q)\right)^{2}} d x_{P} \\
& -\int_{-\infty}^{\infty} \frac{T_{L}(P)\left(\eta_{L}(P)-\eta_{U}(Q)\right)+\delta x^{(-)} \eta_{L}^{\prime}(P)}{\left(\delta x^{(-)}\right)^{2}+\left(\eta_{L}(P)-\eta_{U}(Q)\right)^{2}} d x_{P} .
\end{aligned}
$$

In this expression, it is convenient to define the intermediate functions

$$
\begin{aligned}
T_{U} & =u\left(x, \eta_{U}\right)\left[1+\left(\eta_{U}^{\prime}\right)^{2}\right]-1 \\
T_{L} & =u\left(x, \eta_{L}\right)\left[1+\left(\eta_{L}^{\prime}\right)^{2}\right]-1
\end{aligned}
$$

and the differences

$$
\delta x^{(-)}=x_{P}-x_{Q}, \quad \delta x^{(+)}=x_{P}+x_{Q} .
$$

A similar expression to (4.2) is obtained when point $Q$ is located on the lower interface.

As the solitary wave is left-right symmetric, it is appropriate to exploit this fact in the numerical solution algorithm. It follows that

$$
\begin{aligned}
& \eta_{U}(-x)=\eta_{U}(x) \quad \eta_{L}(-x)=\eta_{L}(x) \\
& u(-x, y)=u(x, y) \quad v(-x, y)=-v(x, y) .
\end{aligned}
$$

When the symmetry relations (4.5) are incorporated into (4.2), the final form of the integral equation, with point $Q$ on the upper interface, becomes

$$
\begin{aligned}
\pi(u-1)_{Q}= & \int_{0}^{\infty} \frac{T_{U}(P)\left(\eta_{U}(P)-\eta_{U}(Q)\right)+\delta x^{(+)} \eta_{U}^{\prime}(P)}{\left(\delta x^{(+)}\right)^{2}+\left(\eta_{U}(P)-\eta_{U}(Q)\right)^{2}} d x_{P} \\
& +C P V \int_{0}^{\infty} \frac{T_{U}(P)\left(\eta_{U}(P)-\eta_{U}(Q)\right)+\delta x^{(-)} \eta_{U}^{\prime}(P)}{\left(\delta x^{(-)}\right)^{2}+\left(\eta_{U}(P)-\eta_{U}(Q)\right)^{2}} d x_{P}
\end{aligned}
$$




$$
\begin{aligned}
& -\int_{0}^{\infty} \frac{T_{L}(P)\left(\eta_{L}(P)-\eta_{U}(Q)\right)+\delta x^{(+)} \eta_{L}^{\prime}(P)}{\left(\delta x^{(+)}\right)^{2}+\left(\eta_{L}(P)-\eta_{U}(Q)\right)^{2}} d x_{P} \\
& -\int_{0}^{\infty} \frac{T_{L}(P)\left(\eta_{L}(P)-\eta_{U}(Q)\right)+\delta x^{(-)} \eta_{L}^{\prime}(P)}{\left(\delta x^{(-)}\right)^{2}+\left(\eta_{L}(P)-\eta_{U}(Q)\right)^{2}} d x_{P} .
\end{aligned}
$$

The intermediate quantities and the differences in this expression are as defined in (4.3) and (4.4). Similarly, if point $Q$ is on the lower interface, the corresponding integral equation is

$$
\begin{aligned}
\pi(u-1)_{Q}= & \int_{0}^{\infty} \frac{T_{U}(P)\left(\eta_{U}(P)-\eta_{L}(Q)\right)+\delta x^{(+)} \eta_{U}^{\prime}(P)}{\left(\delta x^{(+)}\right)^{2}+\left(\eta_{U}(P)-\eta_{L}(Q)\right)^{2}} d x_{P} \\
& +\int_{0}^{\infty} \frac{T_{U}(P)\left(\eta_{U}(P)-\eta_{L}(Q)\right)+\delta x^{(-)} \eta_{U}^{\prime}(P)}{\left(\delta x^{(-)}\right)^{2}+\left(\eta_{U}(P)-\eta_{L}(Q)\right)^{2}} d x_{P} \\
& -\int_{0}^{\infty} \frac{T_{L}(P)\left(\eta_{L}(P)-\eta_{L}(Q)\right)+\delta x^{(+)} \eta_{L}^{\prime}(P)}{\left(\delta x^{(+)}\right)^{2}+\left(\eta_{L}(P)-\eta_{L}(Q)\right)^{2}} d x_{P} \\
& -C P V \int_{0}^{\infty} \frac{T_{L}(P)\left(\eta_{L}(P)-\eta_{L}(Q)\right)+\delta x^{(-)} \eta_{L}^{\prime}(P)}{\left(\delta x^{(-)}\right)^{2}+\left(\eta_{L}(P)-\eta_{L}(Q)\right)^{2}} d x_{P} .
\end{aligned}
$$

The notation CPV in these expressions indicates that the Cauchy Principal Value interpretation is to be given to the integrands that are singular in the limit $P \rightarrow Q$.

The numerical method thus consists of obtaining an approximate solution to the system of equations (2.4)-(2.7) along with the integral equations (4.6) and (4.7) and the amplitude condition (2.2). To do this, a grid of equally-spaced points

$$
0=x_{1}, x_{2}, \ldots, x_{N-1}, x_{N}
$$

is defined, in which the last point $x_{N}$ is taken to be appropriately large. The interval between successive grid points is $\Delta x$. The solution variables at the two interfaces are represented approximately by point values at each of the grid points, and a vector of unknowns

$$
\mathbf{U}=\left[\eta_{1}^{(U)}, \ldots, \eta_{N-1}^{(U)} ; \eta_{1}^{(L)}, \ldots, \eta_{N-1}^{(L)} ; u_{1}^{(U)}, \ldots, u_{N-1}^{(U)} ; u_{1}^{(L)}, \ldots, u_{N-1}^{(L)} ; F\right]^{T}
$$

is established. This vector has $4 N-3$ components.

After an initial guess has been made for the vector $\mathbf{U}$ in (4.8), all the remaining components on both interfaces are then computed. The flow is taken to be uniform far downstream, so that

$$
\eta_{N}^{(U)}=1, \quad \eta_{N}^{(L)}=0, \quad u_{N}^{(U)}=1, \quad u_{N}^{(L)}=1
$$

The derivatives of the two interface elevations are calculated using centred three-point differences. Thus the slope of the upper interface at each mesh point is estimated to be

$$
\left(\eta^{(U)}\right)_{k}^{\prime}=\left(\eta_{k+1}^{(U)}-\eta_{k-1}^{(U)}\right) /(2 \Delta x), \quad k=2, \ldots, N-1
$$

and is zero at the first and last points $k=1$ and $k=N$. The derivatives of the lower 
interface are computed in exactly the same way as (4.10). The two kinematic conditions (2.4) and (2.6) are satisfied directly at each mesh point by computing

$$
v_{k}^{(U)}=u_{k}^{(U)}\left(\eta^{(U)}\right)_{k}^{\prime}, \quad k=1,2, \ldots, N
$$

at the upper interface, and the corresponding vertical velocity component at the lower interface.

An error vector $\mathbf{E}$ of length $4 N-3$ is now defined. Its first $N-1$ components come from the dynamic condition (2.5) at the upper interface, evaluated at all mesh points except the last. The next $N-1$ components are similarly obtained by evaluating (2.7) along the lower interface, at each mesh point except the last one. The next $N-1$ entries in this vector come from the upper integral equation (4.6), with $Q$ chosen to be evaluated at the $N-1$ half-mesh points

$$
x_{k+1 / 2}=\frac{1}{2}\left(x_{k}+x_{k+1}\right), \quad k=1,2, \ldots, N-1
$$

and a further $N-1$ components are obtained by similarly evaluating the lower integral equation (4.7) at the half-mesh points (4.11). Finally, the last element of the error vector $\mathbf{E}$ is derived from the amplitude condition (2.2), and takes the form

$$
E_{4 N-3}=A_{T}+\eta_{1}^{(L)}-\eta_{N}^{(L)}
$$

A damped Newton's method is used to adjust the components of the vector $\mathbf{U}$ in equation (4.8) so as to drive the components of the error vector $\mathbf{E}$ to zero. Convergence to a numerical solution is usually obtained in four or five iterations, and many separate solutions have been computed, typically using $N=201$ points on each interface. This involves Newton's method in a total of 801 variables. The integrals in (4.6) and (4.7) have been evaluated using trapezoidal rule quadrature.

\section{Presentation of results}

Numerical results have been obtained with a variety of density ratios $\gamma_{1}$ and $\gamma_{3}$ in (2.1), and compared with the weakly nonlinear analysis of $\S 3$. To begin, we present solutions for the symmetric case $\gamma_{1}=0.95, \gamma_{3}=1.05$. The small-amplitude solution $A_{T}=0.01$ for this case is shown as a phase-plane plot for the lower interface $\eta_{L}$ in Figure 3. The dashed line is the homoclinic orbit from the weakly nonlinear analysis of $\S 3$, and has been obtained by plotting the first equation in (3.21) against its derivative. The nonlinear result is shown with a solid line, using numerical differentiation as in (4.10). The two results are in close agreement for this low amplitude result.

A large-amplitude solution for this set of density ratios is presented in Figure 4. In this diagram, the amplitude has the value $A_{T}=0.3$, and is close to a maximum limiting profile. This is evident from the figure, since the crests on each interface have become more sharply curved. The limiting wave would involve the formation of a corner at the crest, enclosing the Stokes' angle of $120^{\circ}$ [15], although this precise feature cannot be resolved exactly with the present numerical scheme. The corresponding phase-plane orbits for this case are given in Figure 5, for the lower interface. The dashed line is again the weakly 


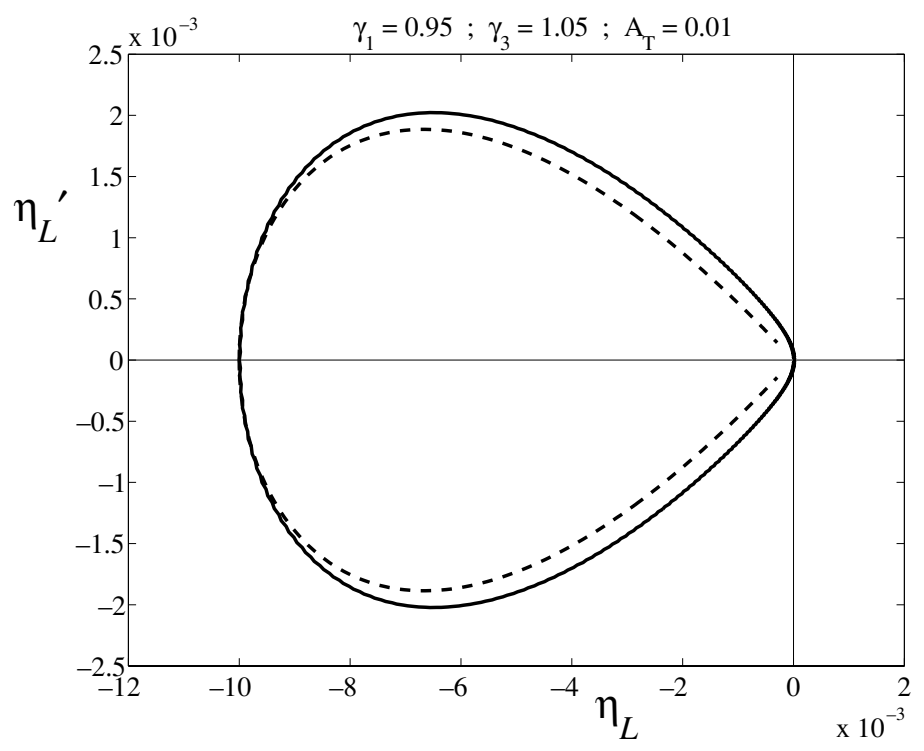

FIGURE 3. Phase-plane plots for the lower interface, for $\gamma_{1}=0.95, \gamma_{3}=1.05$ and amplitude $A_{T}=0.01$. The dashed line is the Korteweg-de Vries solution and the solid line is the nonlinear profile.

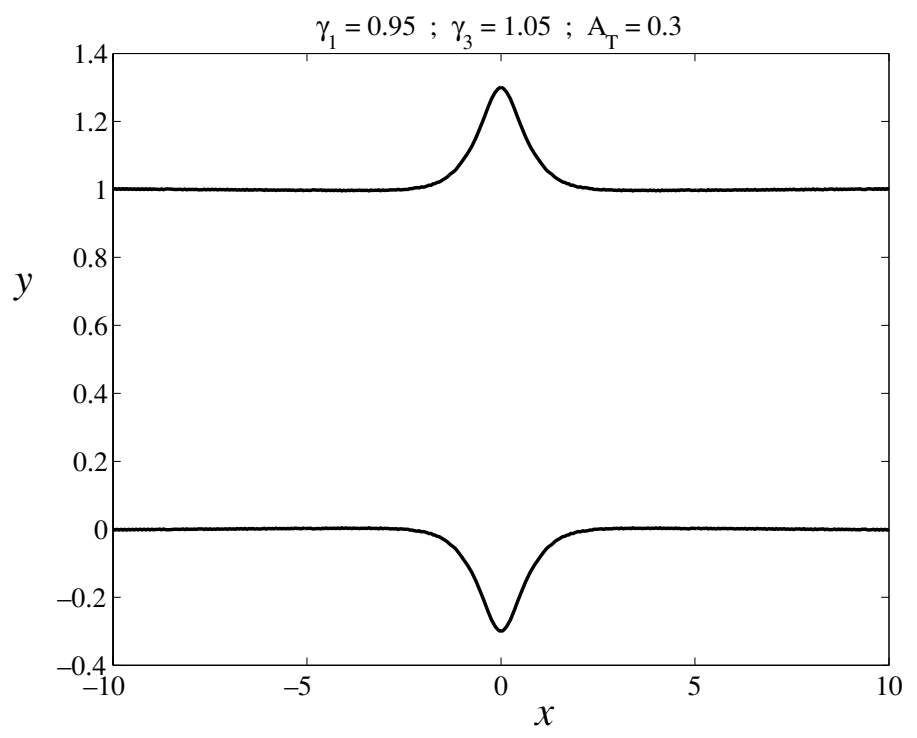

FIGURE 4. Nonlinear solitary wave profile for $\gamma_{1}=0.95, \gamma_{3}=1.05$ and amplitude $A_{T}=0.3$.

nonlinear solution obtained from (3.21) and the solid line is the fully nonlinear numerical result. The two are in broad agreement, although the nonlinear solution is evidently more narrowly pointed near the origin of the phase plane. In addition, some numerical noise due to grid-scale oscillation is present near the origin in Figure 5. 


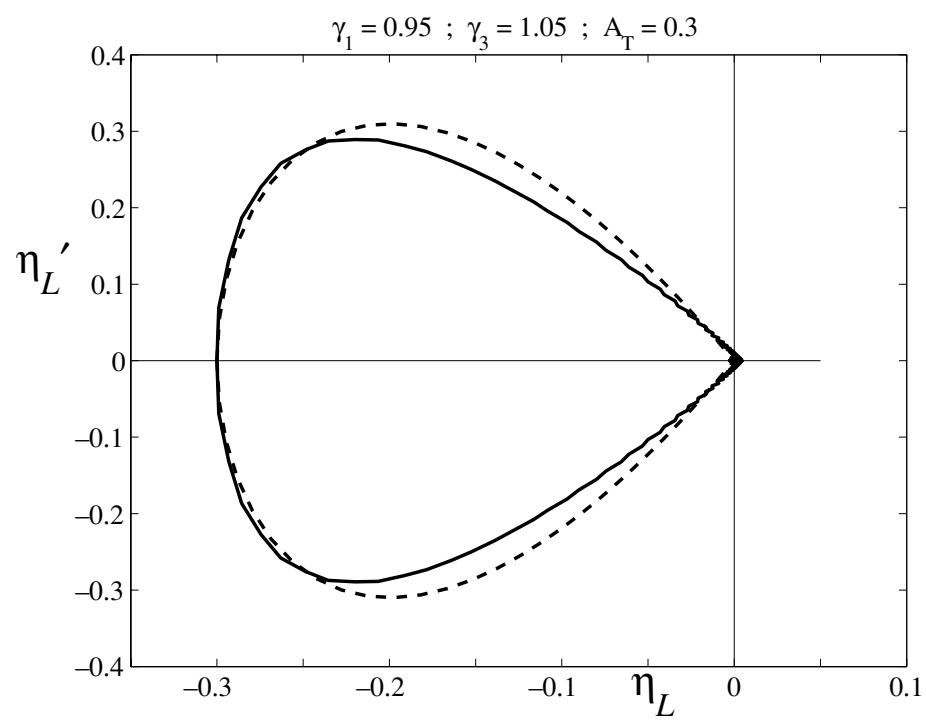

FIGURE 5. Phase-plane plots for the lower interface, for $\gamma_{1}=0.95, \gamma_{3}=1.05$ and amplitude $A_{T}=0.3$, as in Figure 4. The dashed line is the Korteweg-de Vries solution and the solid line is the nonlinear profile.

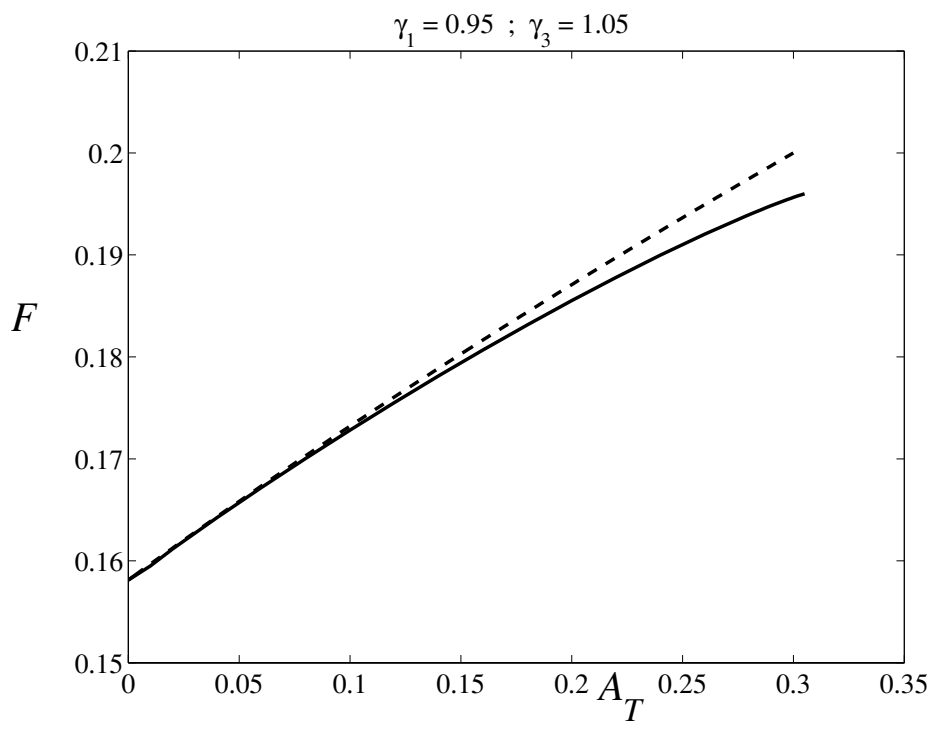

FIGURE 6. Dependence of Froude number on solitary wave amplitude for the case $\gamma_{1}=0.95$, $\gamma_{3}=1.05$. The dashed line is the Korteweg-de Vries solution and the solid line is the nonlinear result.

Figure 6 presents a comparison of the wave-speed (the Froude number in (2.1)) for the weakly nonlinear and numerical solutions, as functions of the wave amplitude $A_{T}$. The two are evidently in close agreement for solitary waves of small to medium amplitude, 


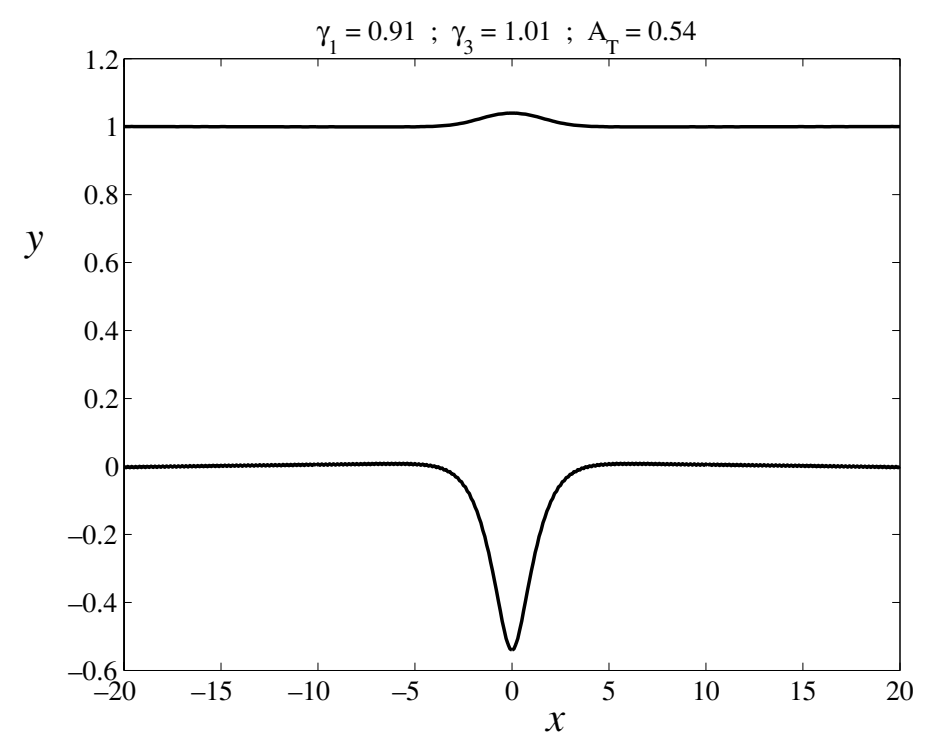

FiguRE 7. Nonlinear solitary wave profile for $\gamma_{1}=0.91, \gamma_{3}=1.01$ and amplitude $A_{T}=0.54$.

and in fact the Korteweg-de Vries result (3.23) gives a reasonable estimate of the speed $F$ over the entire interval of amplitudes $A_{T}$ for which nonlinear solitary waves could be obtained. However, the two curves differ somewhat for large amplitude, and the fully nonlinear solution eventually fails at a value of amplitude slightly larger than $A_{T}=0.3$, when a corner is formed at the crest of the limiting wave profile.

We consider now the situation in which the density jumps across the two interfaces are not symmetrical. The first such case presented here has density ratios $\gamma_{1}=0.91$ and $\gamma_{3}=1.01$. The density jump at the lower interface is thus one tenth of that at the upper interface, so that larger interfacial disturbances are to be expected on the lower surface. It is evident from Figure 7 that this is indeed the case. This solution has been calculated for an amplitude $A_{T}=0.54$, and is close to the maximum limiting wave profile which contains a sharp crest on the lower free surface.

The phase-plane representation of the wave profile in Figure 7 is shown in Figure 8 . The solid line corresponds to the result in Figure 7 for the lower interface $y=\eta_{L}(x)$, and the dashed line is the weakly nonlinear trajectory computed from (3.21). The fully nonlinear profile is narrower near the phase-plane origin than its weakly nonlinear equivalent, and the effects of small grid-scale oscillations are again evident there.

Figure 9 shows the variation of Froude number $F$ with amplitude $A_{T}$ for this case. The fully nonlinear result, drawn with a solid line, agrees well with the predictions of the Korteweg-de Vries analysis (dashed line), for smaller amplitude. The two curves diverge at larger amplitude, and near the maximum wave in the nonlinear case, there is a small region where the wave speed actually decreases slightly, before the limiting profile with an enclosed corner at its crest is formed on the lower interface.

This presentation of results concludes with an investigation of the opposite asymmetry in density jumps at the two interfaces. Figure 10 shows the solitary wave solution for $\gamma_{1}=0.99, \gamma_{3}=1.09$, and for wave amplitude $A_{T}=0.027$. As the density difference at the 


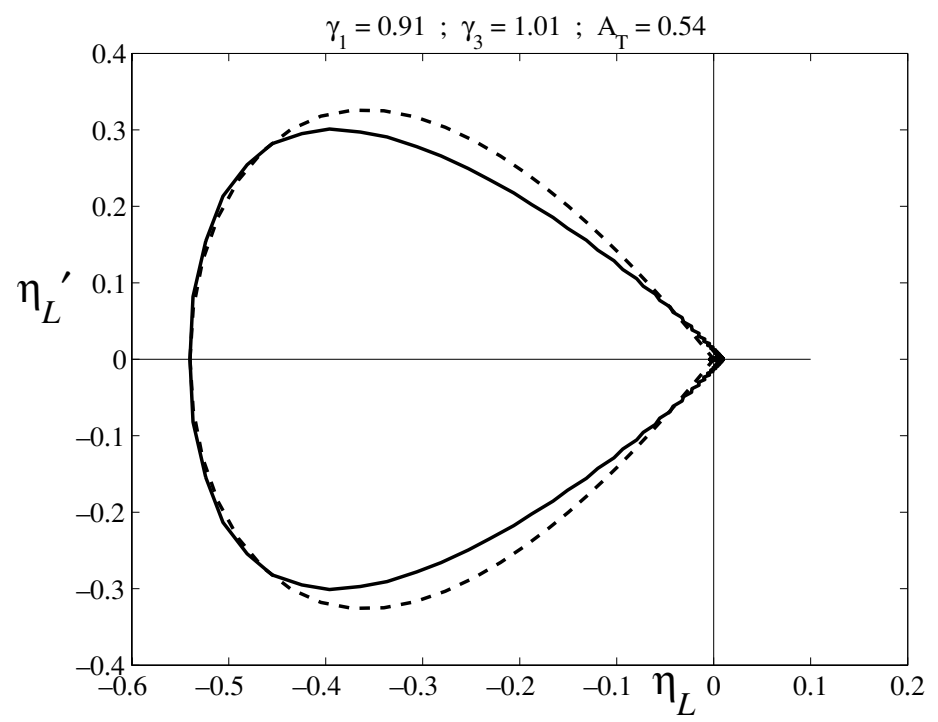

FIGURE 8. Phase-plane plots for the lower interface, for $\gamma_{1}=0.91, \gamma_{3}=1.01$ and amplitude $A_{T}=0.54$, as in Figure 7. The dashed line is the Korteweg-de Vries solution and the solid line is the nonlinear profile.

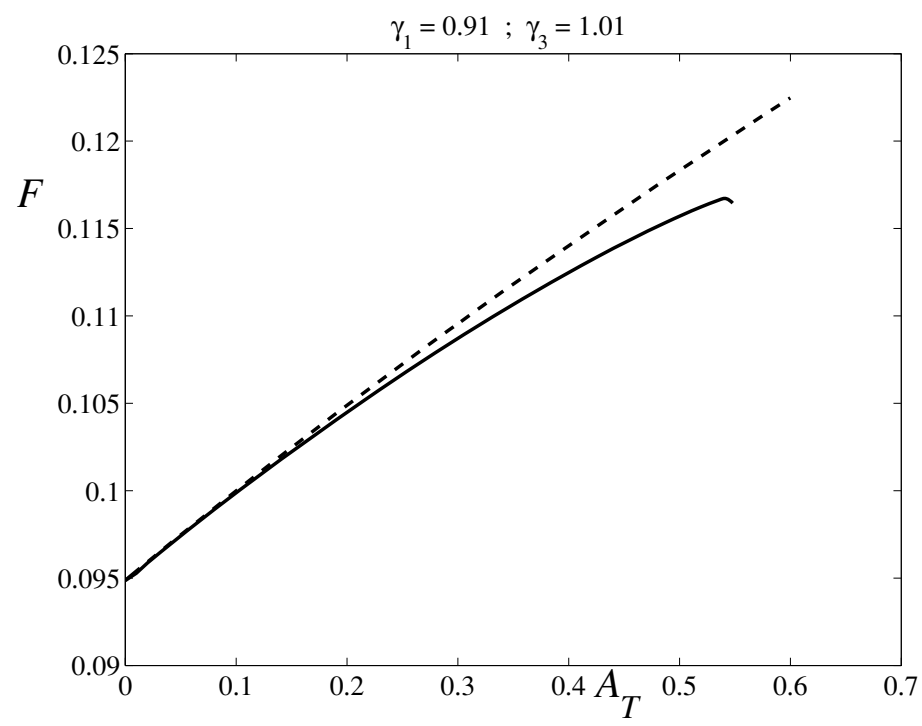

FIGURE 9. Dependence of Froude number on solitary wave amplitude for the case $\gamma_{1}=0.91$, $\gamma_{3}=1.01$. The dashed line is the Korteweg-de Vries solution and the solid line is the nonlinear result.

upper interface is now very small by comparison with that at the lower interface, larger disturbances are to be expected on the upper free surface. The solution in Figure 10 is again close to the maximum wave profile, for which a corner would be formed at the crest of the disturbance on the upper interface, and it is clear that a sharp crest is present 


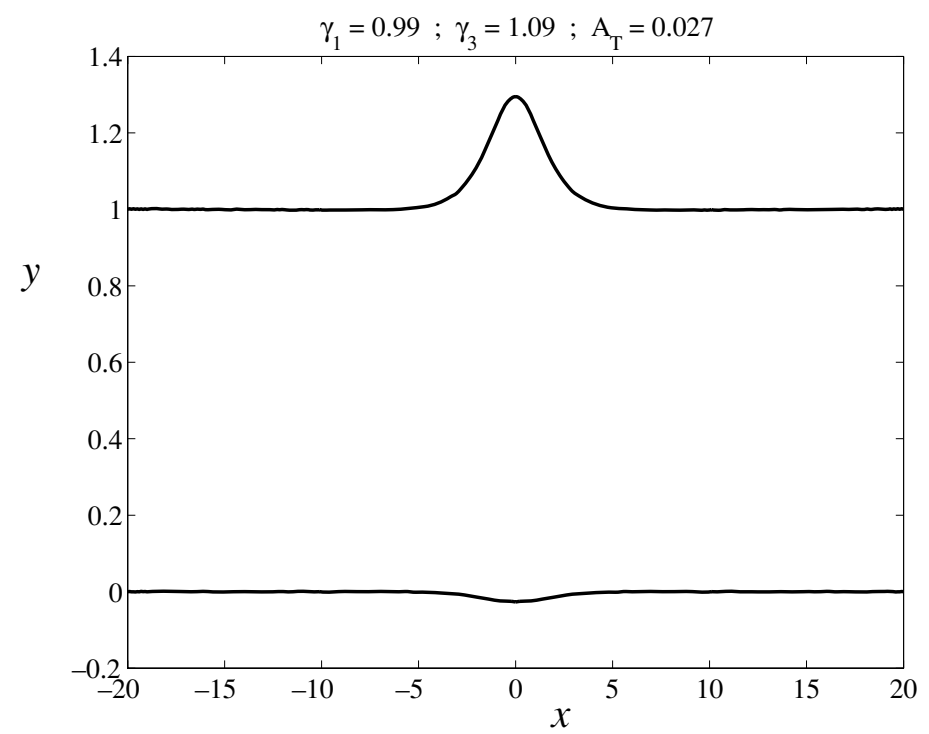

FIGURE 10. Nonlinear solitary wave profile for $\gamma_{1}=0.99, \gamma_{3}=1.09$ and amplitude $A_{T}=0.027$.

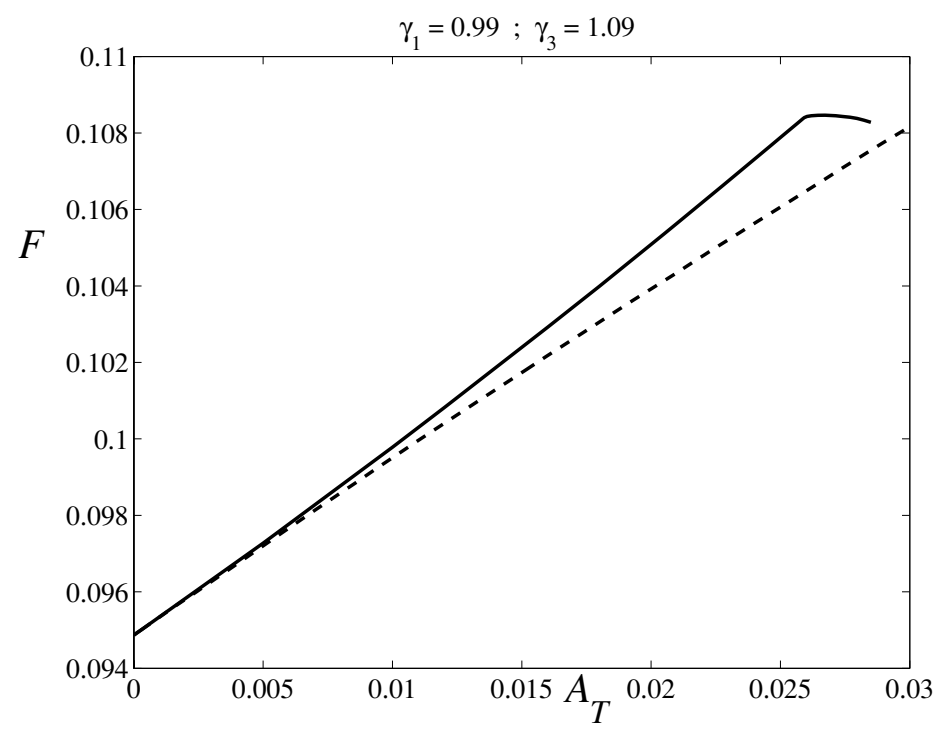

FIGURE 11. Dependence of Froude number on solitary wave amplitude for the case $\gamma_{1}=0.99$, $\gamma_{3}=1.09$. The dashed line is the Korteweg-de Vries solution and the solid line is the nonlinear result.

there. Small grid-scale oscillations may be visible on the lower surface, and these make it more difficult for the nonlinear solution scheme to converge when $A_{T}$ is very close to the wave of maximum amplitude.

The Froude number dependence on amplitude for this case is shown in Figure 11, and is qualitatively similar to previous cases shown in Figures 6 and 9. Agreement between the 
fully nonlinear wave speed and the value (3.23) predicted by Korteweg-de Vries theory is good for small amplitudes, but the two become more dissimilar as the amplitude increases. Near the maximum wave, the nonlinear wave speed again decreases slightly, as is evident from Figure 11.

\section{Conclusion and discussion}

In this paper, solitary waves have been calculated on a moving intrusion layer that is penetrating otherwise stationary stratified fluid of infinite depth. Results have been shown primarily for density ratios pertaining to flows in reservoirs, although similar situations exist in the atmosphere for example. A weakly nonlinear theory was derived, that leads to a Korteweg-de Vries equation for both interfaces. It has been shown that the disturbances to each interface are opposite in sign, so that the soliton occurs as an outwardly directed bulge, for this three-layer system. The fluid speed (3.22) necessarily slows down in that section, as is required to conserve mass.

These predictions have been confirmed using a numerical solution of the fully nonlinear problem, based on an integral-equation technique. The solution profiles and their predicted wave-speeds are in good agreement for small-amplitude waves, but in the nonlinear case, a limiting configuration is ultimately achieved at some maximum amplitude. When the density jumps at the two interfaces are equal, then the system has reflectional symmetry about the centre-line for the moving middle intrusion layer, and in that case the limiting profile involves the formation of corners at the crests of the disturbances on both interfaces simultaneously. Otherwise, for asymmetric density profiles, the limiting wave system has a corner at the crest of the wave on either the upper or the lower interface alone.

As a check on the solitary waves computed in this second paper, we have used the method of part 1 [8], valid for periodic waves of wavelength $\lambda$, to reproduce many of the results presented here. We have computed a large number of waves with very long wavelength $\lambda=200$, and have confirmed that the method in paper 1 can indeed generate the solitary waves produced here. Precisely this approach was adopted by Michallet \& Dias [26] in their study of solitary waves with oscillatory tails.

The numerical technique of this paper is capable of generalization to more complicated soliton flows. It is possible with this physical-plane approach to consider constant vorticity in the fluid layers, although in that case it may be expected that the limiting wave will not necessarily involve a corner enclosing the Stokes angle $120^{\circ}$ at its crests. Instead, there may be overhanging portions in the wave profile, similar to those discussed earlier $[16,17$, $20,21]$, for example. The method presented in $\S 4$ could not compute such waves directly, but can easily be adapted to the task, by re-writing the interfacial boundary conditions in terms of an arclength. When more than one fluid layer is in motion, it is also possible for nonlinear resonances to occur between the flow modes in the different layers, and that more complex flow situation has been discussed by Rusås \& Grue [21] and Michallet \& Dias [26], for example.

\section{Acknowledgements}

This research has been supported in part by Australian Research Council grant number DP0450225. Constructive suggestions from the Referees are gratefully acknowledged. 


\section{References}

[1] Benajmin, T. B. (1968) Gravity currents and related phenomena. J. Fluid Mech. 31, 209-248.

[2] Menta, A. P., Sutherland, B. R. \& KyBA, P. J. (2002) Interfacial gravity currents. II. Wave excitation. Phys. Fluids, 14, 3558-3569.

[3] Sutherland, B. R., Kyba, P. J. \& Flynn, M. R. (2004) Intrusive gravity currents in two-layer fluids. J. Fluid Mech. 514, 327-353.

[4] Manins, P. C. (1976) Intrusion into a stratified fluid. J. Fluid Mech. 74, 547-560.

[5] Wen, X. \& Ingham, D. B. (1992) Flow induced by a submerged source or sink in a three-layer fluid. Comput. Fluids, 21, 133-144.

[6] Papatzacos, P. \& Gustafson, S.-A. (1988) Incompressible flow in porous media with two moving boundaries. J. Comput. Phys. 78, 231-248.

[7] Lamb, H. (1932) Hydrodynamics, 6th ed. Dover.

[8] Forbes, L. K., Hocking, G. C. \& Farrow, D. E. (2005) An intrusion layer in stationary incompressible fluid: Part 1: Periodic waves. Euro. J. App. Math. 16.

[9] Flynn, M. R. \& Sutherland, B. R. (2004) Intrusive gravity currents and internal gravity wave generation in stratified fluid. J. Fluid Mech. 514, 355-383.

[10] Clarke, J. C., Smith, R. K. \& Reid, D. G. (1981) The morning glory of the Gulf of Carpentaria: An atmospheric undular bore. Monthly Weather Review, 109, 1726-1750.

[11] Forbes, L. K. \& Belward, S. (1996) Atmospheric solitary waves: some applications to the Morning Glory of the Gulf of Carpentaria. J. Fluid Mech. 321, 137-155.

[12] NiELD, S. E. \& Woods, A. W. (2004) Effects of flow density on the dynamics of dilute pyroclastic density currents. J. Volcanology and Geotherm. Res. 132, 269-281.

[13] Miles, J. W. (1980) Solitary waves. Ann. Rev. Fluid Mech. 12, 11-43.

[14] Yamada, H. (1957) On the highest solitary wave. Reports of Res. Inst. for Appl. Mech. 5, 53-67.

[15] Hunter, J. K. \& VAnden-Broeck, J.-M. (1983) Accurate computations for steep solitary waves. J. Fluid Mech. 136, 63-71.

[16] Pullin, D. I. \& Grimshaw, R. H. J. (1988) Finite-amplitude solitary waves at the interface between two homogeneous fluids. Phys. Fluids, 31, 3550-3559.

[17] Vanden-Broeck, J.-M. (1994) Steep solitary waves in water of finite depth with constant vorticity. J. Fluid Mech. 274, 339-348.

[18] VANDEN-Broeck, J.-M. \& Dias, F. (1992) Gravity-capillary solitary waves in water of infinite depth and related free-surface flows. J. Fluid Mech. 240, 549-557.

[19] Dias, F. (1994) Capillary-gravity periodic and solitary waves. Phys. Fluids, 6, 2239-2241.

[20] Laget, O. \& Dias, F. (1997) Numerical computation of capillary-gravity interfacial solitary waves. J. Fluid Mech. 349, 221-251.

[21] RusÅs, P.-O. \& Grue, J. (2002) Solitary waves and conjugate flows in a three-layer fluid. Euro. J. Mech. B. Fluids, 21, 185-206.

[22] Dias, F. \& Vanden-BroecK, J.-M. (2002) Generalised critical free-surface flows. J. Eng. Maths. 42, 291-301.

[23] Wehausen, J. V. \& Laitone, E. V. (1990) Surface waves. In: Handbuch der Physik, Vol. 9, edited by S. Flügge, Springer-Verlag.

[24] Whitham, G. B. (1974) Linear and Nonlinear Waves. Wiley.

[25] Grue, J., Friss, H. A., Palm, E. \& Rusi̊s, P. O. (1997) A method for computing unsteady fully nonlinear interfacial waves. J. Fluid Mech. 351, 223-252.

[26] Michallet, H. \& Dias, F. (1999) Nonlinear resonance between short and long waves. Proc. 9th Int. Offshore and Polar Engineering Conf., Brest, France, pp. 193-198. 